\title{
El comercio electrónico en los tratados de libre comercio de México*
}

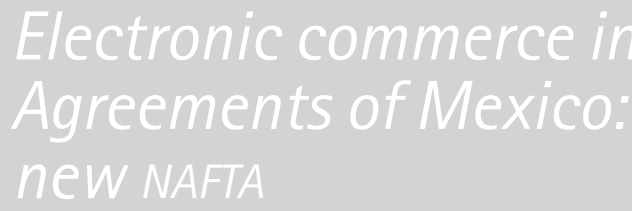

\section{Karen Sigmond**}

\begin{abstract}
RESUMEN
Este artículo iniciará con los antecedentes del desarrollo del régimen del derecho commercial internacional, desde el Acuerdo General sobre Aranceles Aduaneros y Comercio a la Organización Mundial del Comercio. Se definirá al comercio electrónico o E-commerce y se hará una referencia de las tendencias de crecimiento actuales, seguida de la manera en la que se regula esta materia en México. Se estudiará la cobertura del comercio electrónico en los Tratados de Libre Comercio de México, con especial atención en el Acuerdo Transpacífico de Cooperación Económica y la próxima renegociación del Tratado de Libre Comercio de América del Norte, dado el cambio en la política commercial estadounidense bajo la administración de Donald Trump. Finalmente, se abordarán los retos futuros del comercio electrónico y el comercio internacional en un nuevo contexto global.
\end{abstract}

\section{PALABRAS CLAVE}

Comercio internacional, comercio electrónico, tpp, t/can, regulación del E-Commerce.

\begin{abstract}
This article will begin with the background of the development of the international commercial law, from the General Agreement on Tariffs and Trade to the World Trade Organization. Electronic commerce or E-commerce will be defined and a reference will be made to the current growth trends, followed by the way in which this subject is regulated in Mexico. The coverage of e-commerce will be studied in Mexico's Free Trade Agreements, with special attention to the Trans-Pacific Agreement on Economic Cooperation and the forthcoming renegotiation of the North American Free Trade Agreement, given the change in US commercial policy under administration of Donald Trump. Finally, the future challenges of electronic commerce and international trade will be addressed in a new global context.
\end{abstract}

\section{KEYWORDS}

International Commerce, E-Commerce, TPP, NAFTA, Regulation of E-Commerce.

\footnotetext{
*Artículo recibido el 29 de julio de 2017 y aceptado el 23 de agosto de 2017

**Tecnológico de Monterrey, Escuela de Ciencias Sociales y Gobierno, Campus Toluca. (karen.sigmond@itesm.mx) orcid 0000-0003-2123-5469
} 
SUMARIO

1. Introducción

2. Antecedentes del comercio internacional

3. Comercio electrónico, definiciones y números

4. Regulación del comercio electrónico en México

5. Comercio electrónico en los tratados de libre comercio mexicanos

6. Conclusiones

\section{Introducción}

Internet ha revolucionado cada aspecto de nuestras vidas. No solo ha cambiado la manera en que se comparte la información, sino también en cómo llevamos a cabo nuestras actividades cotidianas y satisfacemos nuestras necesidades más básicas, comida y ropa. La era digital ha transformado nuestras vidas: inicialmente, se compraba comida en un mercado, quizá en una tienda local o en un súpermercado, pero ahora se pidie en línea para que sea entregada en nuestros hogares.

La segunda parte de este trabajo describe el desarrollo de las regulaciones en comercio internacional para establecer un antecedente general. La tercera parte define al comercio electrónico e introduce algunas tendencias básicas en su crecimiento. La cuarta parte explica cómo se ha regulado esta materia en México a nivel nacional. La quinta parte discute el comercio electrónico en los tratados de libre comercio (TLC) de México, enfocándose en el Acuerdo Transpacífico de Cooperación Económica (TPP) y analiza cómo puede abordarse este tema en la próxima renegociación del Tratado de Libre Comercio de América del Norte (TLCAN), considerando el posible cambio en la política comercial de los Estados Unidos. Finalmente, la sexta parte ofrece conclusiones relacionadas con el comercio electrónico y un nuevo Tratado de Libre Comercio mexicano.

\section{Antecedentes del comercio internacional}

2.1 Del Acuerdo General sobre Aranceles Aduaneros y Comercio a la Organización Mundial del Comercio

El Acuerdo General sobre Aranceles Aduaneros y Comercio de 1947 (GATT, por sus siglas en inglés) fue el acuerdo internacional que incialmente se enfocó primordialmente en reducir las tarifas entre los países signatarios. Desde entonces, ha evolucionado a lo largo de rondas multilaterales, que han incorporado otros 
temas, como las barreras técnicas al comercio, discriminación de precios, propiedad intelectual e inversiones, por mencionar algunos.

Durante la Ronda Uruguay (1986-1993) se negoció y acordó la Organización Mundial del Comercio (омc). Ésta fue creada para ser el foro de las negociaciones comerciales, administrar los acuerdos, arreglar las diferencias entre sus miembros y dar un espacio de capacitación comercial. De conformidad con el Artículo xXIV del GATT, los miembros pueden pertenecer a zonas de libre comercio y uniones aduaneras, cuyos acuerdos den preferencias que no tienen que ser extendidas a todos los países signatarios del GATT, ahora miembros de la omc. ${ }^{1}$

El comercio internacional se ha desarrollado en dos niveles: en el contexto multilateral (en la omc) y a nivel regional, con el establecimiento de los acuerdos de libre comercio y las uniones aduaneras. Por esta razón, en las décadas pasadas ha habido un cambio a nivel mundal, de un enfoque de negociaciones comerciales multilaterales a acuerdos regionales. Esto ha dado como resultado cerca de seiscientos tratados de libre comercio, uniones aduaneras y otros acuerdos. ${ }^{2}$

Lo anterior surge de la dificultad para lograr que los 164 miembros de la omc acuerden asuntos comerciales que pudieran ser abarcados en un TLC o en una unión aduanera, con menos participantes en la negociación). Consecuentemente, los TLc han incorporado nuevos temas comerciales, tales como el comercio electrónico, que es mucho más lento en el contexto de la omc.

\subsection{El Tratado de Libre Comercio de América del Norte}

Uno de los acuerdos de libre comercio que cambiaron el escenario económico mexicano fue el TLCAN. La terminación de este acuerdo debe ser puesta en contexto. La última parte de la década de los ochenta estuvo caracterizada por un movimiento de libre comercio, tanto a nivel multilateral como regional. $\mathrm{Al}$ nivel multilateral, la mencionada Ronda Uruguay, bajo el GATT, tuvo lugar en 1986. ${ }^{3}$ Ese mismo año, los negociadores estadounidenses y canadienses empezaron a trabajar en un acuerdo de libre comercio entre Estados Unidos y Canadá. Adicionalmente, México se incorporó al GATT e inició una serie de modificaciones legales que pusieron a México en el camino hacia el libre comercio.

\footnotetext{
1 Por ejemplo, los TLC y las Uniones Aduaneras son la excepción al principio de nación más favorecida.

${ }^{2}$ Véase "Reagional Trade Agreements", Organización Mundial del Comercio. [Consulta: 1 de julio, 2017]. Disponible en: https://www.wto.org/english/tratop_e/region_e/region_e.htm

${ }^{3}$ Véase "The Multi-lateral trading system; past, present and future", Organización Mundial del Comercio. [Consulta: 1 de julio, 2017]. Disponible en: www.wto.org/english/thewto_e/whatis_e/inbrief_e/inbr01_e.htm La Ronda Uruguay (1986-1994) resultó en la creación de la Organización Mundial del Comercio (omc).
} 
Los cambios realizados por México a finales de la década de los ochenta y principio de los noventa revirtieron la política económica y comercial que mantuvo por más de cuatro décadas. Pasó de tener una economía cerrada, con un régimen de sustitución de importaciones, a lentamente crear los medios necesarios para sacar al país de una década de crisis, recesión e inflación. ${ }^{4} \mathrm{~A}$ finales de 1989, el entonces presidente, Carlos Salinas de Gortari, presentó al Congreso las reformas que llevaron al movimiento de reprivatización. Para mediados de 1990, los negociadores del TLCAN estaban llevando a cabo las negociaciones entre México y Estados Unidos. Por no quedarse fuera, Canadá se unió al proceso, en septiembre de ese año. El hecho de que México estuviera negociando un acuerdo con sus vecinos del norte envió un mensaje positivo a la comunidad internacional inversionista.

El TLCAN fue un experimento novedoso cuando se firmó. Fue el primer acuerdo de libre comercio entre dos países desarrollados y un país en vías de desarrollo. El texto incluye 22 capítulos y se convirtió en el modelo que México usaría como base para futuros TLc.El artículo 101 establece un área de libre comercio consistente con el Artículo XXIV del GATT. El artículo 102 claramente indica los objetivos del acuerdo, los cuales incluyen eliminar las barreras comerciales y facilitar el movimiento transfronterizo de bienes y servicios, así como promover las condiciones de la competencia justa e incrementar las oportunidades sustanciales de inversión, entre otros.

Es claro que el TLCAN tiene el nivel más bajo de integración económica y sólo involucra el movimiento de bienes y servicios, pues no incluye el movimiento laboral, como en un mercado común. El TLCAN dio la integración económica regional dentro del marco mulitaleral del GATT.

El principal propósito de un TLC es incrementar el comercio y la inversión. Desde 1993, el comercio entre México y Estados Unidos se ha incrementado en $600 \% .{ }^{5}$ México exporta más de $80 \%$ de sus productos a los Estados Unidos y el comercio de los países parte del TLCAN implica 14 millones de empleos, 6 millones exclusivamente gracias a México. ${ }^{6}$

El comercio entre Estados Unidos y México es de más de 1300 millones de dólares al día, casi un millón por minuto. ${ }^{7}$ México es el tercer importador de

\footnotetext{
${ }^{4}$ Véase Sigmond, Karen, Mexican Banking Laws, Evolution into nafta and the Global Economy, Alemania, vdm Verlag, 2008.

${ }^{5}$ Wayne, Earl Anthony, "Why US should treat Mexico as a Vital Partner, not a Punching Bag", The Conversation. [Consulta: 1 de julio, 2017]. Disponible en: http://theconversation.com/why-us-should-treat-mexico-as-a-vitalpartner-not-a-punching-bag-72350

6 "6 million US trade jobs depend on trade with Mexico", Consulmex. [Consulta: 1 de julio, 2017]. Disponible en: https://consulmex.sre.gob.mx/austin/images/pdfs/mapacomercio.pdf

${ }^{7}$ Wayne, Earl Anthony, "Why US should treat Mexico as a Vital Partner, not a Punching Bag", The Conversation.
} 
los productos agrícolas estadounidenses y 57000 empresas estadounidenses le venden a México. Más de 50 \% de la inversión extranjera directa en México proviene de inversionistas estadounidenses. ${ }^{8}$

En virtud de las cifras anteriores, el TLCAN ha sido un éxito. Sin embargo, hay algunos temas, como el comercio electrónico, que no fueron incuidos en él. Por ello, el TPP parece dar la plataforma para nuevos temas comerciales.

\section{Comercio electrónico, definiciones y números}

\subsection{Definiciones}

Para poder comprender qué es el comercio electrónico, vale la pena mencionar algunas definiciones básicas.

El comercio electrónico es definido como la compra y venta de productos o servicios, exclusivamente a través de canales electrónicos. Existen tres ramas principales del comercio electrónico, la forma más conocida es la compra en línea, también conocida como negocio a consumidor (B2C). Aquí, los individuos pueden ordenar diversos productos y pagar por su compra en internet.

Otra categoría de comercio electrónico es de negocio a negocio (B2B), en donde las empresas, como fabricantes, mayoristas o minoristas, pueden intercambiar productos y servicios. La tercera categoría de comercio electrónico involucra transacciones de consumidor a consumidor (C2C), por ejemplo eBay, Etsy u otros sitios similares. ${ }^{9}$ También ha sido establecido que el "comercio electrónico, entendido de manera amplia, es el intercambio de bienes y servicios o el intercambio de información comercial generada entre transmisiones de datos, vía redes de comunicación, como el internet."10

Es indiscutible que este tipo de comercio se ha incrementado radicalmente en años recientes. Más y más individuos y negocios están utilizando internet para propósitos comerciales y conforme esto continúe, las regulaciones también deberán avanzar.

[Consulta: 1 de julio, 2017]. Disponible en: http://theconversation.com/why-us-should-treat-mexico-as-a-vitalpartner-not-a-punching-bag-72350

${ }^{8}$ Wayne, Earl Anthony, "Why US should treat Mexico as a Vital Partner, not a Punching Bag", The Conversation. [Consulta: 1 de julio, 2017]. Disponible en: http://theconversation.com/why-us-should-treat-mexico-as-a-vitalpartner-not-a-punching-bag-72350

9 "E-Commerce in the United States - Statistics and Facts", Statista. [Consulta: 1 de julio, 2017]. Disponible en: https://www.statista.com/topics/2443/us-ecommerce/

${ }^{10}$ Prieto Saldivia, Juan Pablo, "E-Commerce in Chile: Best Practices, Self-Regulation, and Integration", Arizona Journal. Disponible en: http://arizonajournal.org/wp-content/uploads/2016/04/20_Prieto_249.pdf 


\subsection{Cifras y crecimiento desde 1990}

El uso de internet ha revolucionado la manera en que compran los consumidores. Las crecientes cifras de las transacciones comerciales en el mundo digital continúan incrementándose. En especial, podemos observar el aumento en tres áreas diferentes del planeta.

Primero, podemos ver cómo se ha disparado el comercio electrónico en los Estados Unidos. "En 2016, el líder del mercado, Amazon.com generó cerca de US\$46.65 miles de millones de dólares por ventas mediante el comercio electrónico de bienes físicos en Estados Unidos. Las compras en línea son una de las actividades líderes en los Estados Unidos; en 2016, los consumidores estadounidenses gastaron en línea cerca de US\$ 322 miles de millones de dólares.” ${ }^{11}$

Estos números continuarán creciendo, tanto como las preferencias de compra de los consumidores sigan cambiando. Los consumidores ahora pueden sentarse en casa y recibir los productos en su puerta, en lugar de tener que conducir, consumiendo gasolina, a las tiendas o centros comerciales, en donde tendrán que esperar en fila para pagar.

En otras regiones, también puede observarse el aumento en el comercio electrónico. "En América Latina, las transacciones anuales de comercio electrónico fueron de entre US\$60 y \$ US70 miles de millones de dólares, muchos de los cuales caen en la categoría de viajes y entretenimiento, electrónicos y operaciones transnacionales. En una década, el volumen de las transacciones se ha incrementado más de 40 veces." ${ }^{12}$ Las tarjetas de crédito y débito son más comunes ahora. Conforme aumenten los servicios financieros, los consumidores irán cambiando, de usar efectivo, a comprar servicios con tarjetas y en línea. A pesar de la desconfianza inicial hacia las compras en línea, más y más gente está comprando por internet, pues ven los beneficios de no tener que ir físicamete a la tienda para comprar la mercancía.

En otra región, "de acuerdo con un reporte del Gobierno japonés, en 2013, los consumidores japoneses hicieron compras en línea por US\$1.6 miles de milones de dólares de China y Estados Unidos”. ${ }^{13}$ Por otro lado, de acuerdo con

\footnotetext{
11 "Leading e-retailers in the United States in 2016, ranked by e-commerce sales (in billion U.S. dollars)", Statista. [Consulta: 1 de julio, 2017]. Disponible en: https://www.statista.com/statistics/293089/leading-e-retailers-rankedby-annual-web-e-commerce-sales/

12 "Estudio Descriptivo del E-Commerce en Chile y Análisis de Reclamos ante Sernac, E-Commerce en Chile", Sernac. Disponible en: http://www.sernac.cl/wp-content/uploads/2014/08/Reporte_E_Commerce_Reclamos-2013-2014. pdf

${ }^{13}$ Takasugi, Naoshi, "E-Commerce: E-Commerce Law and the Prospects for Uniform E-Commerce Rules on Privacy and Security of Electronic Communications", Arizona Journal. Disponible en: http://arizonajournal.org/wp-content/
} 
la misma fuente, "los consumidores estadounidenses compraron US\$6 miles de millones de dólares en bienes de China o Japón, y los consumidores chinos compraron US\$6.7 miles de millones de dólares en bienes de Japón y Estados Unidos, respectivamente." Más aun, China es el mercado de comercio electrónico más grande de estas tres naciones. "Se espera que la escala de comercio electrónico transfronterizo entre estas tres naciones sumen la cantidad de US\$34 miles de millones de dólares en 2020". ${ }^{14}$

\subsection{Impacto}

Como podemos observar, el comercio electrónico está transformando la manera en que los consumidores globales compran bienes y servicios. Sin importar en qué rincón del planeta resida el consumidor, a través del comercio electrónico puede tener acceso a productos de cualquier parte del mundo. Por otro lado, las empresas tienen acceso a los consumidores de todo el mundo, creando mayores oportunidades de negocios que en la etapa preinternet. No obstante, cada país debe determinar cómo regulará esta actividad y, a pesar de las posibilidades globales de intercambio, la legislación nacional en esta área es multifacética. La regulación nacional incluye muchos ángulos, como prevención de fraudes, protección de consumidores, reglas de contratación, solución de diferencias en línea, por mencionar algunos.

\section{Regulación del comercio electrónico en México ${ }^{15}$}

\subsection{Desarrollo de la legislación nacional basada en modelos internacionales}

La Comisión de las Naciones Unidas para el Derecho Mercantil Internacional (Uncitral) aprobó, en diciembre de 1996, la Ley Modelo de Comercio Electrónico. La incorporación de estas disposiciones al derecho mexicano requirió de la participación de varias instituciones y diversas leyes. Para ello, inicialmente se formó un grupo de organizaciones, conocido como el Grupo EDI, que después se transformó en el Grupo de Trabajo Multisectorial para impulsar la Legislación del Comercio Electrónico (Gilce), el cual emitió la propuesta multisectorial de

\footnotetext{
uploads/2016/04/21_Takasugi_-257.pdf

${ }^{14}$ TAKASUgi, NaOSHI, "E-Commerce: E-Commerce Law and the Prospects for Uniform E-Commerce Rules on Privacy and Security of Electronic Communications", Arizona Journal. Disponible en: http://arizonajournal.org/wp-content/ uploads/2016/04/21_Takasugi_-257.pdf

${ }^{15}$ La autora de este artículo agradece a Eunice Herrera Cuadra por su contribución en esta sección del documento.
} 
reformas legislativas en materia de obligaciones y comercio electrónico. ${ }^{16} \mathrm{El}$ grupo Gilce fue conformado por representantes de diversos organismos, como la Asociación Mexicana de Bancos, la Asociación Mexicana de Estándares para el Comercio Electrónico, la Asociación Mexicana de la Industria de Tecnologías de Información, la Asociación Nacional del Notariado Mexicano, la Secretaría de Comercio y Fomento Industrial (hoy Secretaría de Economía), la Secretaría de Relaciones Exteriores, así como las empresas Infosel (Información Selectiva), Hewlett Packard de México, Oracle de México, Price Waterhouse Coopers y Seguridata Privada.

En 1998, la Organización para la Cooperación y el Desarrollo Económicos (OCDE), a través de su Comité de Políticas del Consumidor, recomendó a los gobiernos, proveedores, consumidores y sus representantes apegarse a directrices, formulación e instrumentación de esquemas autorregulatorios, de protección del consumidor, en materia de comercio electrónico.

En 1999, la Comisión de Comercio de la Cámara de Diputados organizó dos foros de consulta especializada en contratación comercial por medios electrónicos, los cuales concluyeron que era necesario adoptar la Ley Modelo de la Uncitral. ${ }^{17}$ En estos foros intervinieron funcionarios y representantes de la Uncitral, la Cámara Nacional de la Industria Electrónica de Telecomunicaciones e Informática (Canieti), Cecoban Soluciones Interbancarias, el Colegio de Notarios del Distrito Federal, Microsoft México y la Comisión Federal de Competencia Económica, entre otros.

El resultado de todo este proceso fue que, el 29 de mayo de 2000, se publicaron en el Diario Oficial de la Federación las reformas a las leyes y códigos relacionados con la contratación y transacción por medios electrónicos. Éstos son el Código Civil para el Distrito Federal en Materia Común y para toda la República en Materia Federal; el Código Federal de Procedimientos Civiles y la Ley Federal de Protección al Consumidor.

A partir de ese momento, el comercio electrónico en México se encuentra regulado mediante normas que están dirigidas a la protección de los consumidores y a la validez de los contratos comerciales realizados a través de medios electrónicos. Algunas de estas disposiciones jurídicas son la Ley Federal de Telecomunicaciones, la Ley Federal de Protección al Consumidor, el Código

\footnotetext{
${ }^{16}$ Barrios GarRido, Gabriela, Globalización y normatividad del comercio electrónico en México. La importancia de la seguridad jurídica en las transacciones electrónicas. Orden Jurídico, México, Secretaria de Gobernación. [Consulta: 1 de julio, 2017]. Disponible en: http://www.ordenjuridico.gob.mx/Congreso/pdf/105.pdf

17 "Comercio electrónico", Sistema de Información Sobre Comercio Exterior. [Consulta: 1 de julio, 2017]. Disponible en: http://www.sice.oas.org/e-comm/legislation/mex.asp
} 
de Comercio, el Código Civil Federal, el Código Fiscal de la Federación, la Ley Federal del Derecho de Autor y la Ley de la Propiedad Industrial.

Aunque el Código de Comercio y el Código Civil regulan expresamente las operaciones comerciales electrónicas, las otras leyes tienen alguna relación secundaria, como la protección de la propiedad intelectual, los derechos de los consumidores, el servicio de internet, la seguridad en las transacciones bancarias, entre otras funciones.

Así como sucede en el resto del mundo, en México se ha incrementado el uso de internet y el comercio electrónico, por lo cual estas disposiciones jurídicas requieren actualizaciones acordes a esta nueva realidad. Ya han pasado 17 años, de modo que los avances tecnológicos y el crecimiento del comercio en línea hacen imprescindible contar con una sola ley especializada y modernizada.

De igual manera, es preciso actualizar los tratados de libre comercio que están en proceso de renegociación, como es el caso del Tratado de Libre Comercio de América del Norte y el Tratado con la Unión Europea, pues a los temas básicos de regularización del comercio electrónico se le suman cuestiones como la protección de datos personales, ciberseguridad y la portabilidad de datos, por mencionar algunos.

\subsection{Aumento del comercio electrónico en México}

Con base en lo señalado por The 2015 Global Retail E-Commerce Index, en 2015, el volumen de ventas en línea en América Latina alcanzó los 59000 millones de dólares. Según datos de Euromonitor Internacional, la mayor participación pertenece a Brasil (42 \%), seguido por México (18\%), Argentina (12\%), Chile (9\%) y Colombia ( $5 \%) .{ }^{18}$

Según el periódico El Economista, en ese mismo año, el comercio electrónico tuvo un valor de mercado de 257090 millones de pesos en México, lo cual representa un aumento de $59 \%$ desde 2014, cuando su valor fue de 162100 millones de pesos. Asimismo, indica que han aumentado las compras realizadas en línea a un vendedor en el extranjero. La mayoría son importaciones de Estados Unidos, seguidas por países asiáticos. La razón es la variedad en la oferta y el precio. ${ }^{19}$

\footnotetext{
18 "Global Retail E-CommerceKeeps On Clicking", ATKearney. [Consulta: 1 de julio, 2017]. Disponible en: http://www. atkearney.com.mx/consumer-products-retail/e-commerce-index

${ }^{19}$ Riouelme, Rodrigo, "14 datos sobre el comercio electrónico en México", El Economista. [Consulta: 1 de julio, 2017]. Disponible en: http://eleconomista.com.mx/industrias/2016/11/19/14-datos-sobre-comercio-electronico-mexico
} 
Datos de la Asociación Mexicana de Internet (Amipci), citados por El Economista, señalan que el consumidor mexicano sigue prefiriendo realizar las compras de manera tradicional, cuando las tiendas en línea también cuentan con tiendas presenciales. No obstante, existe una tendencia a cambiar esa práctica, particularmente entre la población joven. ${ }^{20}$ De acuerdo con el gobierno mexicano, en 2016, había 37504392 jóvenes de 12 a 29 años, que representan $31.4 \%$ de la población de México. ${ }^{21}$ Estos contribuirán al cambio en la forma de consumo.

\subsection{Nuevos temas que surgen debido al aumento en el comercio electrónico}

Ante el aumento de compras por internet, también se presentan nuevas controversias que requieren soluciones legales adecuadas. Tal es el caso de los sitios de internet que conectan a particulares que venden y compran, como el sitio mexicano Mercado Libre. Aún no ha quedado clara la responsabilidad y el alcance legal de estos sitios, por lo cual se ha llamado la atención de las autoridades responsables; es decir, la Procuraduría Federal del Consumidor (Profeco) y la Secretaría de Economía.

La Profeco únicamente resuelve cuestiones que implican proteger al consumidor desde el punto de vista tradicional; es decir, cuando el vendedor es un negocio, no un particular. Así, la procuraduría no es competente cuando se trata de dos personas que hacen negocios entre sí. ${ }^{22}$ También se presentan lagunas legales cuando un vendedor utiliza los servicios de plataformas en línea como Amazon y, al haber algún incumplimiento, se encuentra desprotegido por falta de regulaciones expresas para esos casos.

Existen discusiones en el Poder Legislativo sobre hacer reformas a la Ley del Consumidor, las cuales permitan clarificar el concepto de relación de consumo entre empresas y terceros. Sin embargo, la Asociación de Internet MX ha señalado riesgos en materia tributaria y un exceso en las regulaciones, las cuales podrían inhibir las inversiones del sector. Por ejemplo, el artículo 9 BIS de la iniciativa de reforma a la Ley Federal de Protección al Consumidor indica que "el proveedor que actúe como intermediario en la comercialización de bienes y

\footnotetext{
${ }^{20}$ Riouelme, Rodrigo, "14 datos sobre el comercio electrónico en México", El Economista. [Consulta: 1 de julio, 2017]. Disponible en: http://eleconomista.com.mx/industrias/2016/11/19/14-datos-sobre-comercio-electronico-mexico 21 "¿Cuántos jóvenes hay en México?", Gob. [Consulta: 1 de julio, 2017]. Disponible en: https://www.gob.mx/gobmx/ articulos/cuantos-jovenes-hay-en-mexico

${ }^{22}$ Ley Federal de Protección al Consumidor. Artículos 1 y 2, definiciones. También ver entrevista al Subprocurador de Telecomunicaciones de la Procuraduria Federal del Consumidor (Profeco), Carlos Ponce Beltrán en El Economista del 4 de abril de 2017.
} 
productos, así como en la prestación de servicios, responderá directamente frente al consumidor por el incumplimiento de las obligaciones contractuales, sin perjuicio de su derecho a repetir contra el comercializador de los bienes y productos, y del prestador de los servicios, o terceros que resulten responsables". ${ }^{23}$

Aun cuando México ocupa el segundo lugar en comercio electrónico en América Latina, queda mucho por aprender y regular. El reto de lograr que la legislación avance a la misma velocidad que la práctica y sus controversias es cada vez mayor.

Por esta razón, son relevantes las negociaciones internacionales de los acuerdos de libre comercio que incluyen el tema de comercio electrónico. Un TLC que incluya esta materia puede dar pie a la armonización de las normas y a una cooperación más profunda entre los países en este tema.

\section{Comercio electrónico en los tratados de libre comercio mexicanos.}

México tiene una red de 12 tratados de libre comercio, lo cual incluye a 46 países, y 32 acuerdos bilaterales de inversión, con 33 países, así como varios acuerdos de cooperación económica. ${ }^{24}$ Es una de las economías más abiertas en el mundo. A pesar del crecimiento entre los socios comerciales, el comercio electrónico no fue incluido en los TLC hasta hace poco. Fue en 2014, en el TLC con Panamá, que finalmente se incluyó el comercio electrónico en el cuerpo del acuerdo. ${ }^{25}$ Por ello, al incluir el comercio electrónico en el Tratado de Asociación Transpacífico (TPP), este tema cubrirá al socio comercial más importante de México: Estados Unidos.

El TPP es un acuerdo entre 12 partes que incluye a Australia, Brunei, Canadá, Chile, Japón, Malasia, México, Nueva Zelanda, Perú, Singapur, Estados Unidos, y Vietnam (la salida de Estados Unidos se discutirá más adelante). En 2016, estas partes cubrieron $40 \%$ del PIB global y aproximadamente $30 \%$ del comercio mundial, tanto en bienes como en servicios. ${ }^{26}$

\footnotetext{
${ }^{23}$ Sánchez Onofre, Julo, "La Ley de Protección al Consumidor se queda corta para el e-commerce", El Economista. [Consulta: 1 de julio, 2017]. Disponible en: http://eleconomista.com.mx/industrias/2017/04/04/ley-proteccion-consumidor-se-queda-corta-e-commerce

24 "México cuenta con 12 tratados de libre comercio", Secretaria de Economia. [Consulta: 1 de julio, 2017]. Disponible en: http://www.gob.mx/se/articulos/mexico-cuenta-con-12-tratados-de-libre-comercio

25 "Tratado de Libre Comercio México-Panamá", Sistema de Informacion Sobre el Comercio Exterior. [Consulta: 1 de julio, 2017]. Disponible en: http://www.sice.oas.org/TPD/MEX_PAN/Draft_MEX_PAN_FTA_s/Index_PDF_09.05.2014_s.asp

${ }^{26}$ Gantz, David, "Simplified Company: The tpp and rcep: Mega-Trade Agreements for the Pacific Rim", Arizona Journal of International and Comparative Law, núm. 33, p. 59.
} 
Para Estados Unidos, bajo la administración de Barak Obama, el TPP era una plataforma de expansión hacia otras naciones asiáticas. De acuerdo con la Oficina de Comercio de Estados Unidos, con Obama el TPP habría nivelado el terreno para los trabajadores y empresas estadounidenses al cortar más de 18000 impuestos diferentes sobre productos estadounidenses. ${ }^{27}$ Usualmente, se le llamaba a esta política comercial "pivote a Asia". ${ }^{28}$ Excluía a China y Rusia, y también estaba en competencia con el área de libre comercio Asia Pacífico (FTAAP, por sus siglas en inglés), respaldada por China y propuesta durante la reunión de Foro de Cooperación Económica Asia-Pacífico (APEC, por sus siglas en inglés) en 2014. ${ }^{29}$

En México, había opiniones encontradas sobre el TPP. Por un lado, algunos indicaban que era una oportunidad para entrar a mercados nuevos y, por el otro, México no quería perder las ventajas competitivas que ya había logrado con el TLCAN, en relación con el mercado estadounidense en particular. ${ }^{30}$ Como sucede en todos los TLC, ha habido ganadores y perdedores, ${ }^{31}$ pero a México este tratado le dio oportunidad de diversificar sus mercados. Además, impulsó el desarrollo de ciertos temas, tales como el comercio electrónico.

El TPP fue visto como el nuevo y más completo acuerdo de libre comercio. Está dividido en 30 capítulos e incluye temas comunes, como trato nacional y acceso a mercados, reglas de origen, inversión, obstáculos técnicos, telecomunicaciones y solución de diferencias, por mencionar algunos. No obstante, también incluye temas que están fuera de TLCAN, como comercio electrónico, trabajo, medio ambiente, pequeñas y medianas empresas, coherencia regulatoria, transparencia y anticorrupción, entre otras. Para efectos de este trabajo, nos centraremos en el capítulo de comercio electrónico.

De acuerdo con el artículo 14.2 del TPP, "Las Partes reconocen el crecimiento económico y las oportunidades proporcionadas por el comercio electrónico, y la importancia de marcos que promueven la confianza de los consumidores en el comercio electrónico y de evitar obstáculos innecesarios para su uso y

\footnotetext{
27 "Electronic Commerce", Office of the United States Trade Representative. [Consulta: 1 de julio, 2017]. Disponible en: https://ustr.gov/sites/default/files/TPP-Chapter-Summary-Electronic-Commerce.pdf

${ }^{28}$ Green, Mike, "The Legacy of Obama's 'Pivot' to Asia", Foreign Policy. [Consulta: 1 de julio, 2017]. Disponible en: http://foreignpolicy.com/2016/09/03/the-legacy-of-obamas-pivot-to-asia/

29 "APEC Summit: Chinese Trade Pact Plan Backed by Leaders", BBC News. [Consulta: 1 de julio, 2017]. Disponible en: http://www.bbc.com/news/world-asia-29999782

30 "Lo bueno y lo malo del tpp para México", Milenio. [Consulta: 1 de julio, 2017]. Disponible en: http://www.milenio. com/negocios/tpp-como_afecta_a_mexico-ftmercados-negocios_0_822517744.html

${ }^{31}$ Grauales, Manuel, "¿Quién gana y quién pierde con el tpp?", Forbes. [Consulta: 1 de julio, 2017]. Disponible en: https://www.forbes.com.mx/quien-gana-y-quien-pierde-con-el-tpp/
} 
desarrollo". Contiene el lenguaje común de los TLc en relación con los aranceles y la no discriminación en productos digitales (artículos 14.3 y 14.4).

El TPP requiere que las partes armonicen su derecho nacional con el internacional; esto es que las lleven a cabo transacciones concordantes con la Ley Modelo de la Uncitral de 1996 sobre Comercio Electrónico, o el Convenio de las Naciones Unidas sobre la Utilización de las Comunicaciones Electrónicas en los Contratos Internacionales (artículo 14.5).

Más aun, requiere que las partes implementen medidas para la protección del consumidor.

De conformidad con el artículo 14.7, las partes deben adoptar o mantener leyes que proscriban actividades comerciales fraudulentas o engañosas que dañen o puedan dañar a los consumidores en línea. El mismo artículo alienta a la cooperación entre las agencias de protección al consumidor de las Partes. Adicionalmente, la información personal del consumidor debe ser protegida por las leyes nacionales, siguiendo los estándares nacionales (artículo 14.8).

El capítulo 14 también alienta a las partes del TPP a moverse al comercio sin consumo de papel (paperless). El artículo 14.9 establece que las partes deben esforzarse para hacer la administración de los documentos comerciales de manera electrónica y que sean legalmente equivalentes a las versiones en papel.

Se promueve tener movimiento de datos transfronterizos e información. El artículo 14.11 aborda las transferencias de información por medios electrónicos y aun cuando reconoce que las partes pueden regular a nivel nacional, las medidas no deben restringir el comercio. El flujo de información es crucial en la economía digital.

La ubicación de las instalaciones informáticas se aborda en el artículo 14.13. En el párrafo 2, se establece que ninguna parte podrá exigir a una persona a usar o ubicar las instalaciones informáticas en el territorio de esa parte, dando flexibilidad de utilizar las instalaciones informáticas ubicadas en cualquier lugar. Los mensajes electrónicos comerciales no solicitados también son regulados en este capítulo. Por primera vez, las partes deben legislar en esta área para proteger a los consumidores de solicitudes no deseadas.

El capítulo de comercio electrónico del TPP llama a la cooperación entre las partes. De conformidad con el artículo 14.15, las partes deben trabajar juntas para asistir a las pequeñas y medianas empresas a intercambiar información y compartir experiencias en reglas, políticas, y cumplimiento de diversos temas. Entre éstos, destacan la protección de la información personal, del consumidor en línea y de mensajes de comercio electrónico no solicitados, así como seguridad, comunicación electrónica, autenticación y gobierno electrónico. La 
cooperación en asuntos de ciberseguridad también es parte de este capítulo (artículo 14.16). Este tema es importante, en virtud del aumento en los ciberataques en esta era digital. ${ }^{32}$

Finalmente, conforme al artículo 14.17, ninguna parte requerirá la transferencia o el acceso al código fuente del programa informático, cuando sea propiedad de una persona de otra parte. Por ello, los proveedores no pueden ser forzados a compartir el código del programa con el gobierno o con otros.

El TPP incorporó compromisos que no existían en los TLC anteriores. Por ejemplo, las partes se comprometieron a tener protección para los consumidores y detener mensajes comerciales no solicitados. También se promovieron el compromiso de ayudar a las pequeñas y medianas empresas. Asimismo, se acordó tener cooperación en materia de amenazas en ciberseguridad y desarrollar capacidad de ciberseguridad, mediante acciones colectivas, lo cual fue un paso hacia la dirección correcta. El lenguaje utilizado en el TPP quizá pueda ser de utilidad para futuros TLC.

Es innegable es que el comercio electrónico ha cambiado la forma en que compran los consumidores y ahora debe ser incluido en los acuerdos comerciales. Las tendencias actuales en Estados Unidos muestran cómo las tiendas físicas crecen a menor ritmo que las tiendas en línea. La National Retail Federation espera que las ventas al menudeo en línea crezcan de 8 a 12 \% en 2017, en tanto se espera que las ventas al menudeo de ladrillo y cemento crezcan solo $2.8 \% .{ }^{33}$ De conformidad con esta misma fuente, las ventas por comercio electrónico generarán entre 427 y 443 miles de millones.

"El comercio electrónico ha ido en aumento en varios años pasados, gracias en parte a titanes de la industria, como Amazon y Alibaba. El comercio electrónico realmente se convertirá en el futuro de las compras al menudeo, ya que casi todo el crecimiento en el sector del menudeo se da en el espacio digital." ${ }^{34}$ Esto no sólo es verdad a nivel nacional, sino también en el contexto global. El comercio electrónico continuará creciendo a lo largo de las fronteras internacionales.

\footnotetext{
${ }^{32}$ Los ciberataques pueden ir contra el gobierno, empresas, tarjetas de crédito de consumo, información financiera, entre otros.

33 "National Retail Federartion Estimates 8-12\% US Eco-Commerce Growth in 2017", Business Insider. [Consulta: 1 de julio: 2017]. Disponible en: http://www.businessinsider.com/national-retail-federation-estimates-8-12-us-e-commerce-growth-in-2017-2017-2

34 "National Retail Federartion Estimates 8-12\% US Eco-Commerce Growth in 2017", Business Insider. [Consulta: 1 de julio: 2017]. Disponible en: http://www.businessinsider.com/national-retail-federation-estimates-8-12-us-e-commerce-growth-in-2017-2017-2
} 
El 20 de enero de 2017, a pesar de haber perdido el voto popular por casi cerca de tres millones, Donald Trump se convirtió en el cuadragésimo quinto presidente de los Estados Unidos. ${ }^{35}$ Muchos observadores esperaban que la retórica de la campaña quedara atrás. Sin embargo, en su primer discurso presidencial, parecía que continuaría con las mismas líneas que demarcó durante su campaña, en relación con la política comercial -básicamente, proteccionismo-.

Durante su discurso anual, mencionó varias frases que reiteraron sus promesas de campaña. Dijo “a partir de este día, sólo será América primero, América primero [...] Debemos proteger nuestras fronteras de los estragos que causan otros países a nuestros productos, robándose nuestras empresas y destruyendo nuestros trabajos". ${ }^{36}$

Posteriormente, indicó que "la protección llevará a una gran prosperidad y fuerza [...] y América empezará a ganar de nuevo, ganar como nunca antes.” Aun más, estableció que "traeremos de regreso nuestros empleos, traeremos de regreso nuestras fronteras [...] seguiremos dos simples reglas: comprar productos estadounidenses y contratar a estadounidenses."

Para los promotores del libre comercio, el mensaje inaugural fue claramente proteccionista en su forma más pura y un retroceso de muchas décadas en la política comercial de Estados Unidos. El 23 de enero de 2017, el presidente Trump firmó una orden ejecutiva para sacar a Estados Unidos del TPP, cumpliendo así con una de sus promesas de campaña y estableciendo una nueva era para los acuerdos comerciales internacionales. Otra promesa de campaña de Trump fue la de construir un muro en la frontera con México. Aunque el muro es un tema que va más allá del alcance de este artículo porque tiene otras implicaciones, tales como migración y seguridad, entre otros, vale la pena mencionarlo, pues el comercio electrónico ahora tiene que lidiar con una nueva realidad en la que las barreras físicas tienen muy poco significado. Los intercambios entre las personas de diferentes países continuarán y algunas áreas, tales como el comercio electrónico, seguirán creciendo, a pesar de las nuevas barreras físicas o comerciales.

Las negociaciones del TLCAN están programadas para iniciar el 16 de agosto de 2017. Al momento de escribir este artículo, los puntos específicos de negociación no se han hecho públicos. No obstante, algunos de estos temas han sido mencionados constantemente en los medios. Dos puntos recurrentes son reglas

\footnotetext{
${ }^{35}$ Krieg, Gregory, "It's official: Clinton swamps Trump in popular vote", cnn Politics. [Consulta: 1 de julio, 2017]. Disponible en: http://edition.cnn.com/2016/12/21/politics/donald-trump-hillary-clinton-popular-vote-final-count/ 36 "Transcript of President Trump's Inauguration Speech", cNBC. [Consulta: 1 de julio, 2017]. Disponible en: http:// www.cnbc.com/2017/01/20/transcript-of-president-trumps-inauguration-speech.html
} 
de origen y comercio electrónico; el primero ya es parte del TLCAN, y el segundo no está incluido en el acuerdo original.

Robert Lighthizer, jefe de la oficina comercial de los Estados Unidos, el US Trade Representative Office, "hizo notar que el acuerdo fue negociado hace más de 23 años y necesita actualizaciones: el TLCAN no contiene regulaciones en materia de comercio electrónico, y carece de los criterios más estrictos en materia medioambiental y de proteccion a la propiedad intelectual de acuerdos más recientes". ${ }^{37} \mathrm{El}$ acuerdo más reciente es el TPP y, por eso, la pregunta es si el capítulo del TPP en comercio electrónico se utilizará como modelo para una posible adición al TLCAN.

Si se utilizara el lenguaje del TPP en el nuevo TLCAN, podemos esperar el trabajo común entre la Profeco y sus equivalentes estadounidense y canadiense. Esto sería de utilidad para los consumidores en el comercio electrónico de Norteamérica. México, Estados Unidos, y Canadá podrían compartir experiencias sobre la regulación de las transacciones entre las diferentes partes en el ciberespacio. Trabajar para entender y armonizar la ley podría llevar a una mayor predictibilidad en la legislación aplicable y, quizá, podría implementarse un mecanismo común de solución de diferencias, en línea.

La cooperación en ciberseguridad entre las partes del TLCAN es más importante que nunca. Al lidiar con seguridad, podemos pensar en seguridad fronteriza y temas como guerra contra el narcotráfico, migración ilegal y otras actividades ilícitas, entre México y Estados Unidos. Asimismo, energía, medio ambiente y protección de las personas contra enfermedades también pueden ser temas de seguridad entre los tres países.

Ahora, todas las áreas tocan internet mediante transacciones financieras que apoyan actividades ilegales y de manera electrónica. Más aun, si hubiera un ciberataque en los puntos de cruce de la frontera, por ejemplo, o cualquier amenaza a la seguridad nacional, relacionada con los sistemas infromáticos, el comercio se detendría. Esto significaría pérdidas de millones de dólares en los tres países.

374 Por ello, es crucial aprender de las lecciones que han dejado seis años de negociaciones del TPP, en espera de ser firmado. El trabajo logrado en materia de comercio electrónico puede sentar las bases para la construcción conjunta de los negociadores del TLCAN.

\footnotetext{
${ }^{37}$ Swanson, Ana, "Trump Administration Formally launches nafa Renegotiation", Washington Post. [Consulta: 1 de julio, 2017]. Disponible en: https://www.washingtonpost.com/news/wonk/wp/2017/05/18/trump-administration-formally-launches-nafta-renegotiation/?utm_term=.adcb89c0b39b
} 


\section{Conclusiones}

La negociación del TLCAN da una oportunidad para incorporar el tema del comercio electrónico. Aun cuando Estados Unidos se salió del TPP, los negociadores pueden modernizar al TLCAN al incluir este importante asunto. Como podemos ver, el contexto multilateral de la omc quizá no sea el ideal para empujar a que avancen las regulaciones en materia de comercio electrónico. Sin embargo, Estados Unidos, México y Canadá tienen la oportunidad de establecer un modelo para abordar el comercio electrónico en futuros acuerdos comerciales. Adicionalmente, esto dará un empuje para que se modernice la legislación mexicana en este tema y, finalmente, se regule en una sola ley que esté armonizada con lo que será el TLCAN 2.0.

\section{Bibliografía}

"6 million US trade jobs depend on trade with Mexico", Consulmex. [Consulta: 1 de julio, 2017]. Disponible en: https://consulmex.sre.gob.mx/austin/images/ pdfs/mapacomercio.pdf"

APEC Summit: Chinese Trade Pact Plan Backed by Leaders”, ввс News. [Consulta: 1 de julio, 2017]. Disponible en: http://www.bbc.com/news/world-asia29999782Barrios Garrido, Gabriela, Globalización y normatividad del comercio electrónico en México. La importancia de la seguridad jurídica en las transacciones electrónicas. Orden Jurídico, México, Secretaría de Gobernación. [Consulta: 1 de julio, 2017]. Disponible en: http://www.ordenjuridico.gob.mx/Congreso/pdf/105.pdf"Comercio electrónico", Sistema de Información Sobre Comercio Exterior. [Consulta: 1 de julio, 2017]. Disponible en: http://www.sice.oas.org/e-comm/legislation/mex.asp

“¿Cuántos jóvenes hay en México?”, Gob. [Consulta: 1 de julio, 2017]. Disponible en: https://www.gob.mx/gobmx/articulos/cuantos-jovenes-hay-en-mexico"E-Commerce in the United States - Statistics and Facts", Statista. [Consulta: 1 de julio, 2017]. Disponible en: https://www.statista.com/topics/2443/ us-ecommerce/“Electronic Commerce”, Office of the United States Trade Representative. [Consulta: 1 de julio, 2017]. Disponible en: https://ustr.gov/ sites/default/files/TPP-Chapter-Summary-Electronic-Commerce.pdf

"Estudio Descriptivo del E-Commerce en Chile y Análisis de Reclamos ante Sernac, E-Commerce en Chile", Sernac. Disponible en: http://www.sernac.cl/ wp-content/uploads/2014/08/Reporte_E_Commerce_Reclamos-2013-2014. pdfGantz, DAVID, "Simplified Company: The TPP and RCEP: Mega-Trade Agreements for the Pacific Rim", Arizona Journal of International and Comparative Law, núm. 33. 
“Global Retail E-CommerceKeeps On Clicking”, ATKearney. [Consulta: 1 de julio, 2017]. Disponible en: http://www.atkearney.com.mx/consumer-products-retail/e-commerce-index

Grajales, Manuel, ¿¿Quién gana y quién pierde con el tpp?”, Forbes. [Consulta: 1 de julio, 2017]. Disponible en: https://www.forbes.com.mx/quien-gana-y-quienpierde-con-el-tpp/Green, Mire, “The Legacy of Obama's 'Pivot' to Asia”, Foreign Policy. [Consulta: 1 de julio, 2017]. Disponible en: http://foreignpolicy. com/2016/09/03/the-legacy-of-obamas-pivot-to-asia/KRIEG, GREGoRY, “It's official: Clinton swamps Trump in popular vote”, cnN Politics. [Consulta: 1 de julio, 2017]. Disponible en: http://edition.cnn.com/2016/12/21/politics/ donald-trump-hillary-clinton-popular-vote-final-count/“Leading e-retailers in the United States in 2016, ranked by e-commerce sales (in billion U.S. dollars)", Statista. [Consulta: 1 de julio, 2017]. Disponible en: https://www. statista.com/statistics/293089/leading-e-retailers-ranked-by-annual-webe-commerce-sales/“

Ley Federal de Protección al Consumidor”, Diario Oficial de la Federación. [Consulta: 1 de julio, 2017]. Disponible en: http://www.diputados.gob.mx/LeyesBiblio/pdf/113_260617.pdf“"

Lo bueno y lo malo del тPP para México”, Milenio. [Consulta: 1 de julio, 2017]. Disponible en: http://www.milenio.com/negocios/tpp-como_afecta_a_mexico-ftmercados-negocios_0_822517744.html“México cuenta con 12 tratados de libre comercio", Secretaria de Economia. [Consulta: 1 de julio, 2017]. Disponible en: http://www.gob.mx/se/articulos/mexico-cuenta-con-12-tratados-de-libre-comercio

“National Retail Federartion Estimates 8-12\% US Eco-Commerce Growth in 2017", Business Insider. [Consulta: 1 de julio: 2017]. Disponible en: http://www. businessinsider.com/national-retail-federation-estimates-8-12-us-e-commerce-growth-in-2017-2017-2

“North American Free Trade Agreement”, nAFTA. [Consulta: 1 de julio, 2017]. Disponible en: www.nafta.sec-alena.org

Prieto Saldivia, Juan Pablo, "E-Commerce in Chile: Best Practices, Self-Regulation, and Integration”, Arizona Journal. Disponible en: http://arizonajournal.org/ wp-content/uploads/2016/04/20_Prieto_249.pdf

"Reagional Trade Agreements”, Organización Mundial del Comercio. [Consulta: 1 de julio, 2017]. Disponible en: https://www.wto.org/english/tratop_e/region_e/ region e.htm

Riquelme, Rodrigo, “14 datos sobre el comercio electrónico en México”, El Economista. [Consulta: 1 de julio, 2017]. Disponible en: http://eleconomista.com.mx/ industrias/2016/11/19/14-datos-sobre-comercio-electronico-mexico

SÁnchez Onofre, Julio, "La Ley de Protección al Consumidor se queda corta para el e-commerce”, El Economista. [Consulta: 1 de julio, 2017]. Disponible en: http://eleconomista.com.mx/industrias/2017/04/04/ 
ley-proteccion-consumidor-se-queda-corta-e-commerce SIGMOND, KAREN, Mexican Banking Laws, Evolution into NAFTA and the Global Economy, Alemania, vDM Verlag, 2008.

Swanson, Ana, "Trump Administration Formally launches nAFTA Renegotiation", Washington Post. [Consulta: 1 de julio, 2017]. Disponible en: https://www. washingtonpost.com/news/wonk/wp/2017/05/18/trump-administration-formally-launches-nafta-renegotiation/?utm_term=.adcb89c0b39b

TAKasugi, NaOShI, "E-Commerce: E-Commerce Law and the Prospects for Uniform E-Commerce Rules on Privacy and Security of Electronic Communications", Arizona Journal. Disponible en: http://arizonajournal.org/wp-content/ uploads/2016/04/21_Takasugi_-257.pdf

“Transcript of President Trump's Inauguration Speech”, cNBc. [Consulta: 1 de julio, 2017]. Disponible en: http://www.cnbc.com/2017/01/20/transcript-of-president-trumps-inauguration-speech.html

Wayne, Earl Anthony, "Why US should treat Mexico as a Vital Partner, not a Punching Bag", The Conversation. [Consulta: 1 de julio, 2017]. Disponible en: http://theconversation.com/why-us-should-treat-mexico-as-a-vital-partner-not-a-punching-bag-72350 